\title{
The rice RCN1/OsABCG5 mutation is associated with root de- velopment in response to nutrient shortage
}

\author{
An-na Ureshi ${ }^{1}$, Shuichi Matuda ${ }^{1}$, Emiko Ohashi ${ }^{1}$, Kazumitsu Onishi ${ }^{1}$, Itsuro Takamure ${ }^{2}$ and \\ Kiyoaki Kato ${ }^{1}$ \\ ${ }^{1}$ Department of Crop Science, Obihiro University of Agriculture and Veterinary Medicine, Nishi 2-11 Inada, \\ Obihiro, Hokkaido, 080-8555, Japan \\ ${ }^{2}$ Graduate School of Agriculture, Hokkaido University, Kita 9 Nishi 9, Kita-ku, Sapporo, Hokkaido, 060-8589, \\ Japan \\ Corresponding author: K. Kato, E. mail: kiyoaki@obihiro.ac.jp, Phone: +81-155-49-5477, FAX: +81-155-49-5593
}

Received on October 11, 2011; Accepted on June 19, 2012

\begin{abstract}
Plants constantly sense changes in their environment. When nutrient elements are scarce, plants often allocate a greater proportion of their biomass to the root system. In the present study, we characterized the early root development of rice reduced culm number ( $r c n)$ mutants under standard growth conditions. Short lateral root length was consistent in five $r c n$ mutants, and the remaining root traits varied between the rcn mutants. Subsequently, we characterized the effects of rcn1 mutation in response to nutrient shortage. Root development, involving the elongation of seminal, crown, and lateral roots and branching of the lateral roots, was promoted in wild type in response to nutrient shortage. However, in rcn1 mutants, crown root elongation was constant and lateral root branching was reduced in response to nutrient shortage. The present results indicate that RCN1/OsABCG5 is associated with root development in response to nutrient shortage.
\end{abstract}

Keywords: Nutrient shortage, RCN1/OsABCG5, reduced culm number, rice, root architecture

\section{Introduction}

Plants constantly sense changes in their environment and when nutrient elements are scarce, plants often allocate a greater proportion of their biomass to the root system. It is known that deficiencies in essential macronutrients (nitrogen, phosphorus, potassium, and magnesium) result in an accumulation of carbohydrates in leaves and roots, which modifies the root-shoot biomass ratio (Reviewed by Hermans et al.
2006). To date, ion transport mechanisms and the biochemical pathways affected by mineral deficiencies have been well elucidated (Epstein et al. 2005); however, the molecular and physiological events responsible for sensing and signaling mineral resource limitations and their ultimate effects on plant development and biomass allocation are not well understood.

The rice reduced culm number 1 (rcn1-2) mutant, reported as a low tillering mutant (Takamure et al. 1989, Yasuno et al. 2007, 2009), delayed flowering by more than 40 days, and reduced shoot and root dry weights in comparison with wild type under nutrient-limiting conditions. This suggests that ren1-2 mutation reduces plant development for sensing nutrient shortage and/or reduces nutrient uptake from the soil under low-nutrient conditions (Ariyaratna et al. 2011). We determined that RCN1 encodes a novel protein in the ATP-binding cassette protein $G$ (ABCG) subfamily, also known as the white-brown complex (WBC) subfamily, OsABCG5 (OsWBC5) (Yasuno et al. 2009). The molecular function of RCN1/OsABCG5 remains to be elucidated. The present study was conducted to determine if RCN1/OsABCG5 is essential for root development in response to nutrient shortage. In addition to $r c n l$, ten other genes with mutations leading to reduced culm number have been reported (Takamure et al. 1989, Takamure and Kinoshita 1993, Takamure 1994, Tang et al. 2001, Li et al. 2003, Jiang et al. 2006, Yasuno et al. 2007, Ariyaratne et al. 2009); however the root phenotypes of $r c n$ mutants have not been described yet. In the present study we conducted two experiments. In the first experiment, we characterized the early root development of $r c n 1$ mutant in comparison with the four other independent reduced culm number

Ureshi A, Matuda S, Ohashi E, Onishi K, Takamure I, Kato K 2012 The rice RCN1/OsABCG5 mutation is associated with root development in response to nutrient shortage. Plant Root 6: 28-35. doi:10.3117/plantroot.6.28

Copyrights 2012, Plant Root (JSRR), www.plantroot.org 
mutants $(r c n 2, r c n 4, r c n 5$, and $r c n 6)$ in rice from the japonica background under normal conditions. In the second experiment, we examined whether rcnl mutation affects early root development in response to nutrient shortage.

\section{Materials and Methods}

\section{Experiment 1}

We examined the rice (Oryza sativa L. ssp. japonica) cultivar Shiokari and five rice mutants originating from Shiokari; S-97-61 (rcn1-2), N-174 (rcn2), $\mathrm{N}-187$ (rcn4-2), N-185 (rcn5), and N-186 (rcn6). These five $r c n$ mutations are at independent loci, based on an allelism test (Takamure et al. 1989, Takamure and Kinoshita 1993, Takamure 1994). All seeds were surface sterilized and incubated in the dark at $30^{\circ} \mathrm{C}$ for 2 days to allow germination. Germinated seeds were transferred to plastic boxes $(26 \times 21 \times 0.5 \mathrm{~cm})$ containing Murashige and Skoog (MS) medium (Murashige and Skoog 1962) (without sugar, $\mathrm{pH} 5.8,1.3 \%$ agar) at $\times 1(1-\mathrm{MS})$ concentration and were grown under a regime of $16 \mathrm{~h}$ of light $(350$ $\mu \mathrm{mol} / \mathrm{m}^{2} \mathrm{~s}$ ) at $28^{\circ} \mathrm{C}$ and $8 \mathrm{~h}$ of darkness at $24^{\circ} \mathrm{C}$. The lower parts of the plastic boxes were covered with black paper to keep the roots continuously in the dark. The following observations were recorded daily from Day 5 to Day 12: the number of leaves (leaf stage), shoot length, lengths of the seminal and crown roots, the number of crown roots, and the number and length of lateral roots on the seminal root. The density of the lateral roots on the seminal root axis was determined by dividing the number of lateral roots by the length of the seminal root for each plant. Lateral root lengths were measured with a ruler using $0.5 \mathrm{~mm}$ increments.

\section{Experiment 2}

Shiokari and $r c n 1-2$ seeds were surface sterilized and incubated in the dark at $30^{\circ} \mathrm{C}$ for 2 days to allow germination. Germinated seeds were transferred to plastic boxes $(26 \times 21 \times 0.5 \mathrm{~cm})$ containing MS medium (Murashige and Skoog 1962) (without sugar, $\mathrm{pH} 5.8,1.3 \%$ agar $)$ at $\times 1(1-\mathrm{MS}), \times 0.5(0.5-\mathrm{MS})$, and $\times 0(0-\mathrm{MS})$ concentrations and were grown as mentioned in Experiment 1. At 10 days after germination we recorded the same traits as Experiment 1.

\section{Statistical analysis}

For the analysis of root traits in Shiokari and the five $r c n$ mutants, one-way analysis of variance (ANOVA) was performed for each trait in Experiment 1. Least significant difference (LSD) was used for comparison

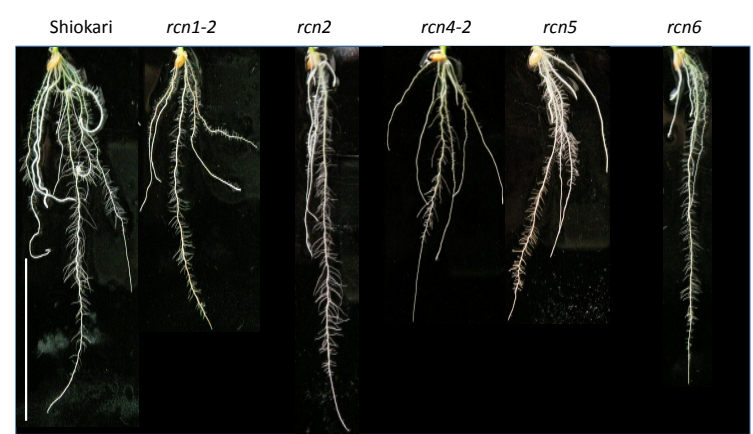

Fig. 1. Root morphology of wild-type Shiokari and the five $r c n$ mutants 12 days after planting. Plants were grown on 1-MS agar medium (without sugar, $\mathrm{pH} 5.8,1.3 \%$ agar). Scale bar represents $5 \mathrm{~cm}$.

among the mean values of the six strains. Two-way ANOVA was performed to analyze the effect of genotype and nutrient treatments on root traits in Experiment 2. Means were separated by the LSD test if the $F$-test indicated a significant difference $(\mathrm{P}<0.05)$. All statistical analysis was conducted using PASW $^{\mathbb{R}}$ Statistics 18 (SPSS, Inc.).

\section{Results}

\section{Root architecture of the five rcn mutants}

Seedlings were grown on 1-MS agar medium to compare root morphology of the different $r c n$ mutants. The root architecture of 12-day-old $\mathrm{rcn}$ seedlings and the wild-type Shiokari is shown in Fig. 1. Shoot growth was delayed in all $r c n$ mutants by 1 or 2 days compared to that in the wild type (Table 1). To eliminate the effect of leaf stage on root development, we measured root architecture when the seedlings were at the 3-leaf stage (Table 1). rcn1-2 seedlings had significantly shorter lateral roots and a larger lateral root density in comparison with Shiokari. rcn2 seedlings had a significantly longer seminal root, larger number of lateral roots and shorter total crown and lateral root length in comparison with Shiokari. $r c n 4-2$ seedlings had significantly shorter shoots, seminal and lateral roots, fewer number of lateral roots and lower lateral root density in comparison with Shiokari. rcn5 seedlings had significantly shorter seminal roots, total crown and lateral roots, higher lateral root density and a fewer number of crown roots in comparison with Shiokari. rcn6 seedlings had significantly shorter total crown and lateral root length and a fewer number of crown roots.

Among all the characteristics tested in the five $r c n$ mutants, the reduction in lateral root length was the most evident. To characterize the effect of $r c n$ genes on lateral root elongation, the kinetics of lateral root 
Table 1. Morphological analysis of seedlings of wild-type Shiokari, $r c n 1-2, r c n 2, r c n 4, r c n 5$, and $r c n 6$ at the 3-leaf stage.

\begin{tabular}{lcccccccc}
\hline Genotype & $\begin{array}{c}\text { Days to 3rd } \\
\text { leaf stage }\end{array}$ & $\begin{array}{c}\text { Shoot length } \\
(\mathrm{cm})\end{array}$ & $\begin{array}{c}\text { Seminal root } \\
\text { length } \\
(\mathrm{cm})\end{array}$ & $\begin{array}{c}\text { Crown root } \\
\text { number }\end{array}$ & $\begin{array}{c}\text { Total crown } \\
\text { root's length } \\
(\mathrm{cm})\end{array}$ & $\begin{array}{c}\text { Lateral root } \\
\text { number }\end{array}$ & $\begin{array}{c}\text { Lateral root } \\
\text { length } \\
\left(\mathrm{mm}^{2}\right)\end{array}$ & $\begin{array}{c}\text { Lateral root } \\
\text { density } \\
\left(\mathrm{cm}^{-1}\right)\end{array}$ \\
\hline Shiokari & $9.0 \pm 0.0 \mathrm{~d}$ & $13.6 \pm 0.6 \mathrm{ab}$ & $10.4 \pm 0.8 \mathrm{~b}$ & $5.0 \pm 1.1 \mathrm{a}$ & $18.1 \pm 5.0 \mathrm{a}$ & $113.2 \pm 18.3 \mathrm{~b}$ & $5.1 \pm 0.7 \mathrm{a}$ & $10.9 \pm 1.9 \mathrm{~b}$ \\
$r c n 1-2$ & $10.5 \pm 0.5 \mathrm{~b}$ & $12.5 \pm 1.2 \mathrm{bc}$ & $9.8 \pm 1.0 \mathrm{bc}$ & $5.0 \pm 1.1 \mathrm{a}$ & $15.9 \pm 4.1 \mathrm{a}$ & $131.2 \pm 26.5 \mathrm{ab}$ & $2.6 \pm 0.6 \mathrm{c}$ & $13.3 \pm 2.2 \mathrm{a}$ \\
$r c n 2$ & $10.7 \pm 0.5 \mathrm{~b}$ & $12.3 \pm 1.4 \mathrm{c}$ & $11.6 \pm 0.8 \mathrm{a}$ & $3.8 \pm 1.7 \mathrm{ab}$ & $9.2 \pm 4.6 \mathrm{c}$ & $149.5 \pm 29.1 \mathrm{a}$ & $3.6 \pm 0.8 \mathrm{~b}$ & $12.9 \pm 2.2 \mathrm{ab}$ \\
$r c n 4-2$ & $10.2 \pm 0.9 \mathrm{bc}$ & $10.9 \pm 0.9 \mathrm{~d}$ & $6.7 \pm 0.1 \mathrm{~d}$ & $4.7 \pm 1.5 \mathrm{a}$ & $13.4 \pm 4.9 \mathrm{ab}$ & $50.0 \pm 14.3 \mathrm{c}$ & $2.1 \pm 0.3 \mathrm{c}$ & $7.5 \pm 2.2 \mathrm{c}$ \\
$r c n 5$ & $11.2 \pm 0.9 \mathrm{a}$ & $14.1 \pm 0.9 \mathrm{a}$ & $9.1 \pm 1.0 \mathrm{c}$ & $3.0 \pm 0.7 \mathrm{~b}$ & $10.9 \pm 1.6 \mathrm{bc}$ & $136.0 \pm 11.5 \mathrm{ab}$ & $2.7 \pm 0.9 \mathrm{c}$ & $15.0 \pm 0.6 \mathrm{a}$ \\
$r c n 6$ & $9.8 \pm 1.0 \mathrm{c}$ & $12.7 \pm 1.3 \mathrm{bc}$ & $10.6 \pm 0.1 \mathrm{ab}$ & $2.8 \pm 1.0 \mathrm{~b}$ & $9.7 \pm 2.1 \mathrm{bc}$ & $139.3 \pm 22.4 \mathrm{ab}$ & $4.0 \pm 1.0 \mathrm{~b}$ & $13.2 \pm 2.0 \mathrm{ab}$ \\
\hline
\end{tabular}

To evaluate morphological characteristics, the seedlings were grown on 1-MS agar medium (without sugar, $\mathrm{pH} 5.8,1.3 \%$ agar). Data are the average for six plants $( \pm \mathrm{SD})$.

Different letters indicate significant differences $(\mathrm{P}<0.05)$ between genotypes.

elongation were compared (Fig. 2). The lateral roots of Shiokari and rcn6; ren1-2, rcn2, and $r c n 5$; and rcn4-2 seedlings began elongating at 5, 6 , and 7 days after germination (DAG), respectively. The lateral root elongation of $r c n 1-2, r c n 5$, and $r c n 6$; Shiokari and $r c n 2$; and $r c n 4-2$ seedlings almost ceased at 7, 8, and $12 \mathrm{DAG}$, respectively. Based on the kinetics of lateral root elongation, the five $r c n$ mutants were divided into three groups. In the first group, the elongation period was shortened, as represented by rcn6 seedlings, in which the elongation rate was similar to Shiokari at between 5 and 6 DAGs and elongation ceased at 7 DAG. In the second group, the onset was delayed and the elongation period was

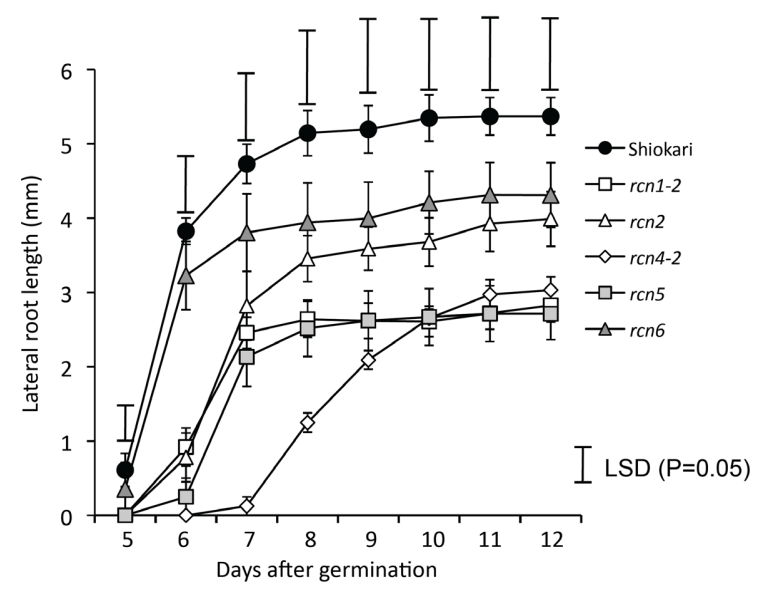

Fig. 2. Kinetic comparison of lateral root elongation between wild-type Shiokari and the five $r c n$ mutants. The length of lateral roots emerging from the region between $2 \mathrm{~cm}$ and $4 \mathrm{~cm}$ from the base of the seminal root is represented. Plants were grown on 1-MS agar medium (without sugar, $\mathrm{pH}$ 5.8, 1.3\% agar). Each value represents the average for six plants $( \pm \mathrm{SE})$. shortened, as represented by $r c n 1-2, r c n 2$, and $r c n 5$. In the third group, the onset was delayed until 7 DAG and the elongation rate was reduced, as represented by $r c n 4-2$ seedlings.

\section{Response to nutrient shortage in rcn1-2 mutant}

We tested the possibility that $r c n 1-2$ affects root development in response to nutrient shortage using 10-day-old seedlings. The two-way ANOVA test indicated significant interaction between genotype and treatment in seminal root length, crown root number, length of the longest crown root, total crown root length, lateral root number and lateral root density (Table 2). In Shiokari seedlings, no differences were detected between 1-MS and 0.5-MS medium in all root traits tested (Fig. 3). When 0-MS media was used as the nutrient shortage condition on Shiokari seedlings, it affected shoot elongation and the development of three root-types; seminal, crown, lateral roots. Shiokari and $r c n 1-2$ seedlings showed reduced shoot length on 0-MS medium in comparison with 1-MS or 0.5-MS (Fig. 3A). Shiokari seedlings on 0-MS medium significantly promoted the elongation of seminal, the longest crown and total crown length compared with 1-MS by factors of 1.8, 2.3, and 1.6 times, respectively. For $r(n 1-2$, the significant difference between 0 - and 1-MS was detected in seminal root length. In $r c n 1-2$ seedlings, the elongation of seminal root length in 0 -MS medium was 1.5 times larger than that in 1-MS medium, and the longest crown, total crown and lateral root lengths in 0 -MS medium tended to be greater than those in 1-MS medium. Crown root number in Shiokari seedlings was significantly reduced by $34 \%$ on 0 -MS medium compared to 1-MS, whereas the crown root number in rcn1-2 on 0.5 -MS medium was significantly increased compared to that on 1-MS medium (Fig. 3C). On 0-MS medium, Shiokari seedlings 
Table 2. Two-way ANOVA statistics of the effect of ren1-2 genotype on shoot length, seminal root length, crown root number, length of the longest crown root, total crown root length, lateral root number on seminal roots, lateral root density on seminal roots, and lateral root length on seminal roots of 10-day-old seedlings sorted by MS medium concentration.

\begin{tabular}{|c|c|c|c|c|c|}
\hline Traits & Variation & $d f$ & MS & $F$ & $P$ \\
\hline \multirow[t]{4}{*}{ Shoot length } & Genotype & 1 & 5160.0 & 19.6 & $<0.001$ \\
\hline & Treatment & 2 & 3034.2 & 11.5 & $<0.001$ \\
\hline & Genotype $\times$ Treatment & 2 & 78.4 & 0.3 & $\mathrm{~ns}$ \\
\hline & Erorr & 30 & 263.9 & & \\
\hline \multirow[t]{4}{*}{ Seminal root length } & Genotype & 1 & 9834.0 & 17.8 & $<0.001$ \\
\hline & Treatment & 2 & 18098.1 & 32.8 & $<0.001$ \\
\hline & Genotype $\times$ Treatment & 2 & 3788.1 & 6.9 & $<0.01$ \\
\hline & Erorr & 30 & 551.9 & & \\
\hline \multirow[t]{4}{*}{ Crown root number } & Genotype & 1 & 4.7 & 2.7 & ns \\
\hline & Treatment & 2 & 13.0 & 7.5 & $<0.01$ \\
\hline & Genotype $\times$ Treatment & 2 & 12.7 & 7.3 & $<0.01$ \\
\hline & Erorr & 30 & 1.7 & & \\
\hline \multirow{4}{*}{$\begin{array}{l}\text { Length of the longest } \\
\text { crown root }\end{array}$} & Genotype & 1 & 26136.1 & 105.4 & $<0.001$ \\
\hline & Treatment & 2 & 11166.6 & 45.0 & $<0.001$ \\
\hline & Genotype $\times$ Treatment & 2 & 9373.7 & 37.8 & $<0.001$ \\
\hline & Erorr & 30 & 247.9 & & \\
\hline \multirow[t]{4}{*}{ Total crown root length } & Genotype & 1 & 326041.0 & 42.9 & $<0.001$ \\
\hline & Treatment & 2 & 75472.7 & 9.9 & $<0.001$ \\
\hline & Genotype $\times$ Treatment & 2 & 61062.8 & 8.0 & $<0.01$ \\
\hline & Erorr & 30 & 7605.5 & & \\
\hline \multirow[t]{4}{*}{ Lateral root number } & Genotype & 1 & 21904.0 & 40.4 & $<0.001$ \\
\hline & Treatment & 2 & 2149.8 & 4.0 & $<0.05$ \\
\hline & Genotype $\times$ Treatment & 2 & 17557.3 & 32.3 & $<0.001$ \\
\hline & Erorr & 30 & 542.9 & & \\
\hline \multirow[t]{4}{*}{ Lateral root density } & Genotype & 1 & 0.0 & 0.0 & ns \\
\hline & Treatment & 2 & 90.3 & 9.7 & $<0.001$ \\
\hline & Genotype $\times$ Treatment & 2 & 57.1 & 6.1 & $<0.01$ \\
\hline & Erorr & 30 & 9.3 & & \\
\hline \multirow[t]{4}{*}{ Lateral root length } & Genotype & 1 & 66.0 & 49.9 & $<0.001$ \\
\hline & Treatment & 2 & 8.7 & 6.6 & $<0.01$ \\
\hline & Genotype $\times$ Treatment & 2 & 3.9 & 3.0 & ns \\
\hline & Erorr & 30 & 1.3 & & \\
\hline
\end{tabular}

showed a 1.6 times increase in lateral root number with a constant density, whereas $r c n 1-2$ showed a reduction in both lateral root number and density by $36 \%$ and $60 \%$, respectively, compared to $1-\mathrm{MS}$ (Fig. $3 \mathrm{~F}, 3 \mathrm{G})$. As a res ult, the lateral root number and density of $r c n 1-2$ se edlings were significantly lower than those of Shiokari seedlings on 0-MS medim.

The developmental characteristics in 10-day-old plants of the lateral roots along the seminal root grown on 1-, 0.5-, and 0-MS media were examined in detail. The relationship between the number and length of lateral roots for each 2-cm segment of the 

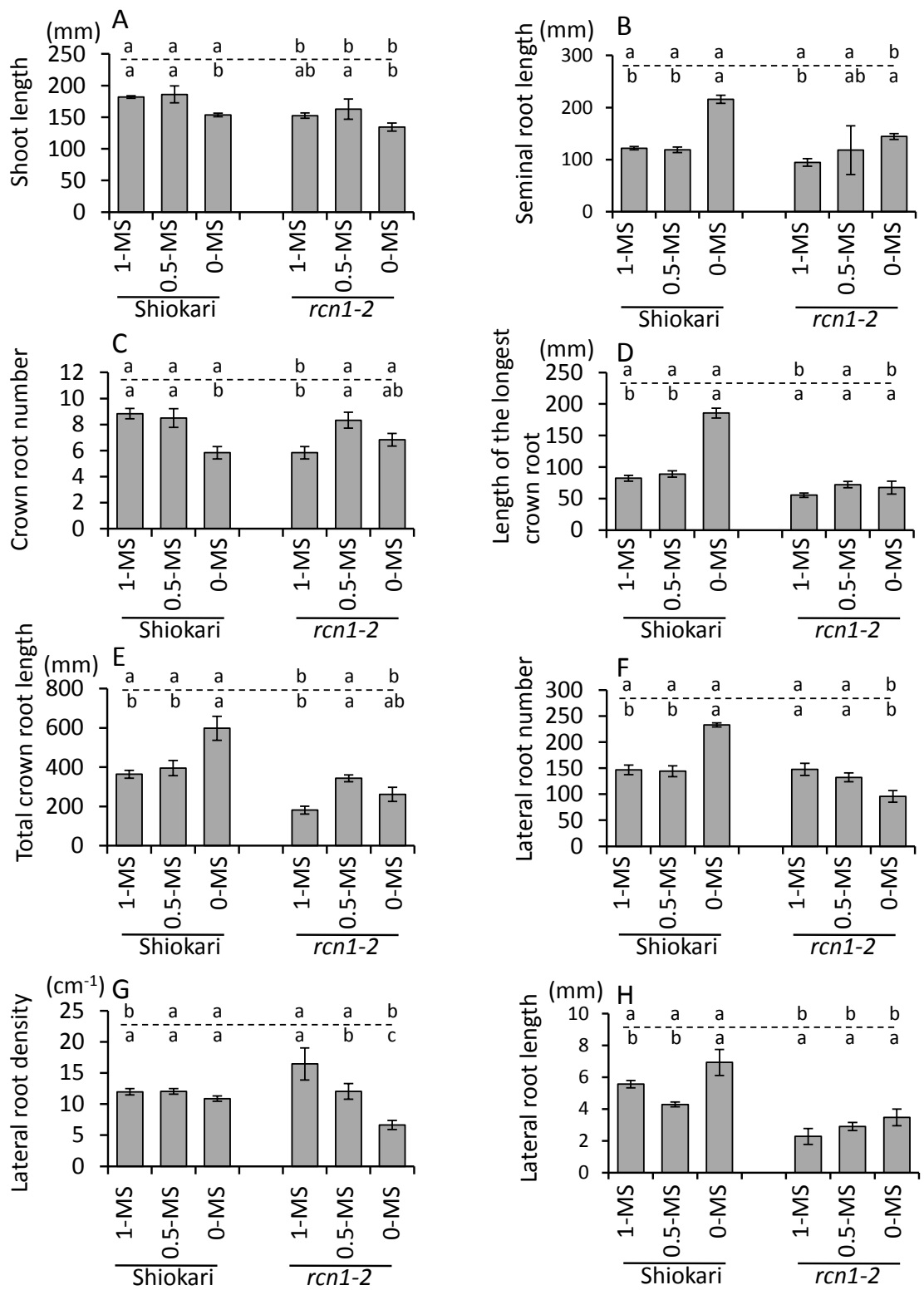

Fig. 3. Shoot length (A), seminal root length (B), crown root number (C), length of the longest crown root (D), total crown root length $(\mathrm{E})$, lateral root number on seminal roots $(\mathrm{F})$, lateral root density on seminal roots $(\mathrm{G})$, and lateral root length on seminal roots $(\mathrm{H})$ of 10-day-old seedlings. Each value is the average for six plants $( \pm \mathrm{SE})$. For each strain, different letters under the dotted line indicate significant differences $(\mathrm{P}<0.05)$ between nutrient conditions. For each nutrient condition, different letters on the dotted line indicate significant differences $(P<0.05)$ between strains.

seminal root axis is shown in Fig. 4. Each genotype has a similar number of lateral roots in each segment on 1- and 0.5-MS media (Fig. 4A, 4B, 4C, 4D). In Shiokari seedlings, the lateral root number per segment remained constant on 0-MS medium, with a maximum number of 25-30 roots at the basal part of seminal roots, between the base and $16 \mathrm{~cm}$. In the same region of $r c n 1-2$ seedlings, the lateral root number was reduced to less than 20 roots. Combining the effects of shortened seminal roots and reduced lateral root branching per segment resulted in a reduction in the total lateral root number of $r \mathrm{cn} 1-2$ seedlings on 0-MS medium. Both the minimum and maximum length of the lateral roots per seminal root segments in both genotypes increased on 0-MS medium in comparison with 0.5-MS and 1-MS medi um. On 0-MS medium, the lateral root elongation in Shiokari increased in the section of the seminal root between $6 \mathrm{~cm}$ and $16 \mathrm{~cm}$. In $\mathrm{rcn} 1-2$, there were few differences in lateral root length among the MS treatments. 
Shiokari

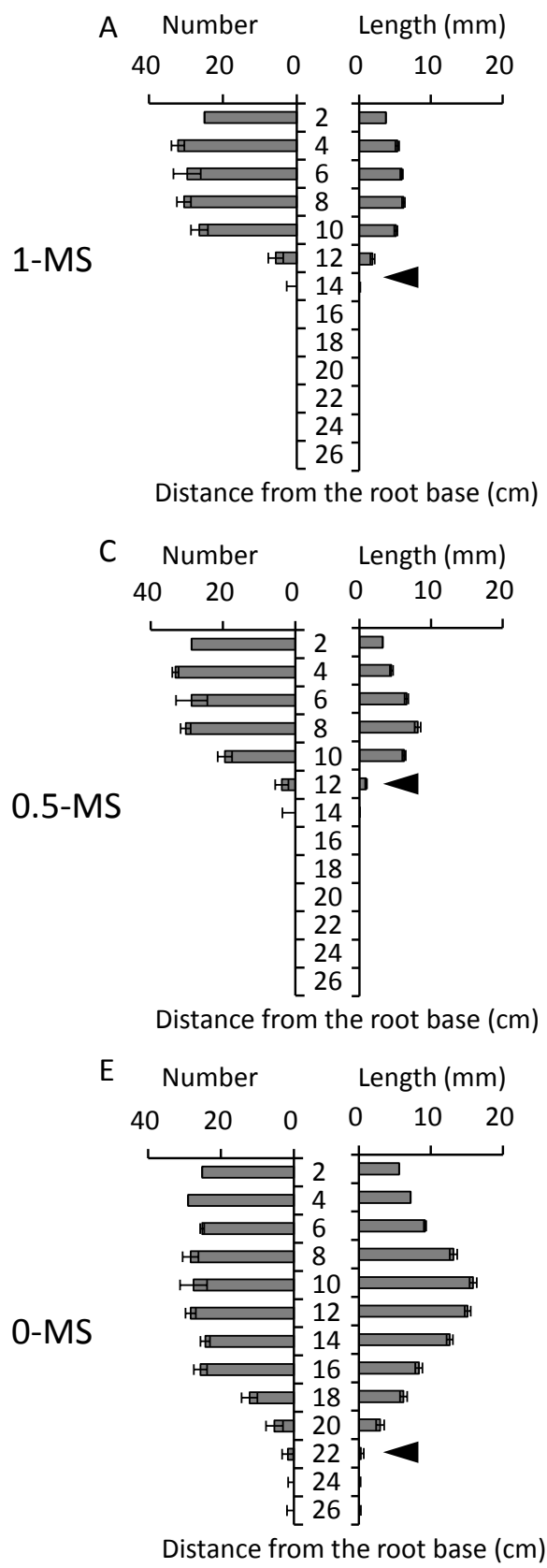

ren1-2

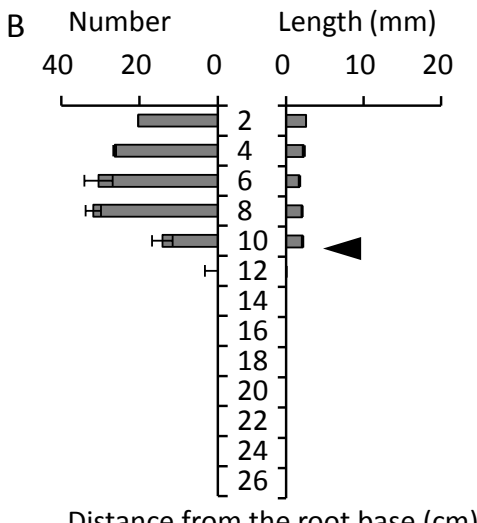

Distance from the root base $(\mathrm{cm})$

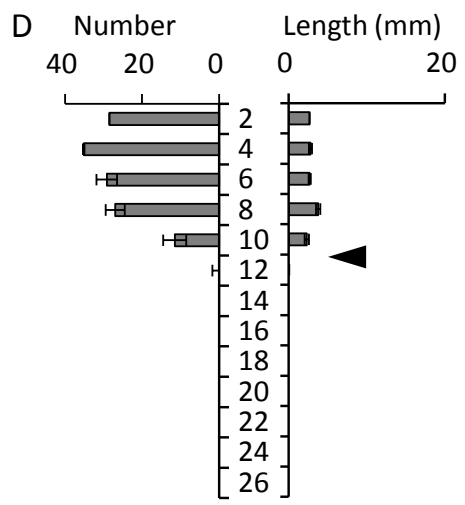

Distance from the root base $(\mathrm{cm})$

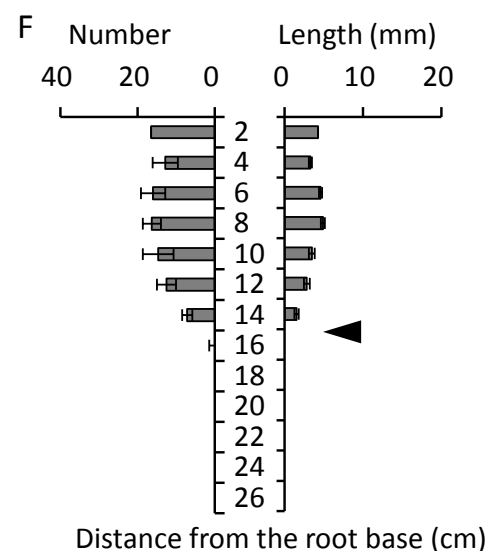

Fig. 4. Lateral root number (left) and length (right) in each 2-cm segment of the seminal root of 10-day-old Shiokari (A, C, E) and $\operatorname{rcn} 1-2(\mathrm{~B}, \mathrm{D}, \mathrm{F})$ grown on 1-, 0.5- and 0-MS medium (without sugar, $\mathrm{pH} 5.8,1.3 \%$ agar). Each value is the average for six plants $( \pm \mathrm{SE})$. Black triangles represent the positions of the root tip.

\section{Discussion}

The present study showed the variable effects of the $r c n$ gene on early root development, independent of shoot growth and node number. Among these root characteristics, the lateral roots of the five $r c n$ mutants were consistently shorter than those of the wild-type Shiokari (Fig. 2, Table 1). Lateral roots play important roles in providing an absorptive area for nutrients and water, as well as anchoring the plant in the soil (Banoc et al. 2000, Ma et al. 2001, Bailey et al. 2002, Kamoshita et al. 2004). Our study suggests that shoot branching during growth is influenced by the same genetic system that affects root branching. However, we could not eliminate the possibility that nutrient limitation due to limited root 
development also reduced shoot branching in $r c n$ mutants.

It is clear that plants have evolved sophisticated pathways for sensing and responding to changes in various external nutrients as well as their own internal nutrient status. Shortage of nutrients in soil rapidly slows plant shoot growth, whereas root growth is often less inhibited, thereby increasing the relative surface area available for ion absorption (Chapin 1990). Nitrogen and phosphorus deficiencies result in an increase in the root-shoot biomass ratio (Hermans et al. 2006). The present study showed that nutrient shortage reduced shoot length and promoted elongation of seminal, crown, and lateral roots as well as the emergence of lateral roots in Shiokari seedlings (Fig. 3). Under nutrient limiting conditions, the crown root number was reduced, whereas total crown root length increased; showing that promoting the elongation of fewer crown roots resulted in an increase of total crown root length in Shiokari. In Shiokari under nutrient limiting conditions, lateral root density was maintained, seminal root elongation was promoted, and lateral root length increased, which in turn resulted in an increase in total lateral root length. In contrast, the degree of elongation of all three root types tended to be lower and the emergence of lateral roots were significantly reduced in rcn 1-2 mutants under nutrient shortage conditions. The present evidence suggests that the RCN1/ OsABCG5 mutation is associated with sensing nutrient shortage or the morphogenesis in root development in response to nutrient shortage. The reduction in root development in response to nutrient shortage may inhibit root functions in taking up water and nutrients from the soil, sensing abiotic and biotic stresses, and serving as an anchor that supports shoots. In a future study, we need to clarify the effect that this poor root development in ren 1-2 on its root functions under nutrient shortage conditions.

Nutrient deficiency was reported to stimulate abscisic acid (ABA) biosynthesis in roots and intensify the root-to-shoot ABA signal (Chapin 1990, Peuke et al. 1994, 2002, Saab et al. 1990, Jeschke et al. 1997, Jeschke and Hartung 2000, Schraut et al. 2005). Accumulation of ABA leads to a higher root-shoot biomass ratio in nutrient-deficient plants (Saab et al. 1990, Vystoskaya et al. 2008). Vystoskaya et al. (2008) reported that ABA accumulates in the root apices of nutrient-deficient plants and is involved in maintaining root growth. In addition to ABA, cytokinin-mediated signaling seems to control plant development under nitrogen deficiency, and coordinated changes in cytokinin, auxin, and ethylene concentrations may reprogram development under phosphorus deficiency (Hammond et al. 2004, Ticconi and Abel 2004, Franco-Zorrilla et al. 2004).
RCN1/OsABCG5 mRNA accumulates not only in leaf primordia of main and axillary shoots and in the vascular cells and leaf epidermis of older leaves, but also in the crown root primordia, endodermis, pericycle, and stele in the roots (Yasuno et al. 2009). In future studies, we need to clarify which element and phytohormone is associated with RCN1/ OsABCG5 function in roots. Finally, identification of the remaining $r c n$ genes and clarification of their functions at the molecular level could explain whether $r c n$ genes control the growth of both lateral shoots and roots.

\section{Acknowledgements}

We are grateful to Dr. Jun Abe of the University of Tokyo, Dr. Yoshiaki Inukai and Dr. Mikio Nakazono of Nagoya University for their valuable suggestions. This work was supported by Grant-in-Aid for Scientific Research (C) (21580002) from the Ministry of Education, Culture, Sports, Science and Technology, the Program for Promotion of Basic and Applied Researches for Innovations in Bio-oriented Industry (BRAIN), and the Global Center of Excellence Program, Ministry of Education, Culture, Sports, Science and Technology (to S.M.).

\section{References}

Ariyaratne M, Takamure I, Kato K 2009 Shoot branching control by reduced culm number 4 in rice (Oryza sativa L.). Plant Sci. 176: 744-748.

Ariyaratna M, Takamure I, Kato K 2011 Plant architecture and its responses to high planting density and low fertilizer of reduced culm number mutants in rice (Oryza sativa L.). J. Plant Breed. Crop Sci. 3: 114-119.

Bailey PHJ, Currey JD, Fitter AH 2002 The role of root system architecture and root hairs in promoting anchorage against uprooting forces in Allium cepa and root mutants of Arabidopsis thaliana. J. Exp. Bot. 53: 333-340.

Banoc DM, Yamauchi A, Kamoshita A, Wade LJ, Pardales Jr. JR 2000 Genotypic var iations in response of lateral root development to fluctuating soil moisture in rice. Plant Prod. Sci. 3: 335-343.

Chapin III FS 1990 Effects of nutrient deficiency on plant growth: evidence for a centralized stress-response system. In: Davies WJ, Jeffcoat B, Eds., Importance of root to shoot communication in the responses to environmental stress. The British Society of Plant Growth Regulations, Monograph No.21, Parchments, Oxford, pp. 135-148.

Epstein E, Bloom AJ 2005 Mineral nutrition of plants: principles and perspectives, 2nd edn. Sinauer Associates, Inc. Publishers. Sunderland, MS.

Franco-Zorrilla JM, Gonzalez E, Bustos R, Linhares F, Leyva A, Paz-Ares J 2004 The transcriptional control of plant responses to phosphate limitation. J. Exp. Bot. 55: 28 5-293.

Hammond JP, Broadley MR, White PJ 2004 Genetic responses to phosphorus deficiency. Ann. Bot. 94: 323-332.

Hermans C, Hammond JP, White PJ, Verbruggen N 2006 
How do plants respond to nutrient shortage by biomass allocation? Trends Plant Sci. 11: 1360-1385.

Jeschke WD, Hartung W 2000 Root-shoot interactions in mineral nutrition. Plant Soil 226:57-69.

Jeschke WD, Peuke AD, Pate JS, Hartung W 1997 Transport, synthesis, and catabolism of abscisic acid (ABA) in intact plants of castor bean (Ricinus communis L.) under phosphate deficiency and moderate salinity. J. Exp. Bot. 48: 1737-1747.

Jiang H, Guo BL, Xue DW, Zeng DL, Zhang GH, Dong GJ, Gu MH, Qian Q 2006 Genetic analysis and gene-mapping of two Reduced-Culm-Number mutants in rice. J. Integr. Plant Biol. 48: 341-347.

Kamoshita A, Rodriguez R, Yamauchi A, Wade LJ 2004 Genotypic variation in response of rainfed lowland rice to prolonged drought and rewatering. Plant Prod. Sci. 7: 406-420.

Li XY, Qian Q, Fu Z, Wang YH, Xiong GS, Zeng DL, Wang $X$, Liu X, Teng S, Fujimoto H, Yuan M, Luok D, Han B, Li J 2003 Control of tillering in rice. Nature 422: 618-621.

Ma JF, Goto S, Tamai K, Ichii M 2001 Role of root hairs and lateral roots in silicon uptake by rice. Plant Physiol. 127: 1773-1780.

Murashige T, Skoog F 1962 A revised medium for rapid growth and bioassays with tobacco tissue cultures. Physiol Plant 15: 473-497.

Peuke AD, Jeschke WD, Hartung W 1994 The uptake and flow of C, N and ions between roots and shoots in Ricinus communis L. III. Long-distance transport of abscisic acid depending on nitrogen nutrition and salt stress, J. Exp. Bot. 45: 741-747.

Peuke AD, Jeschke WD, Hartung W 2002 Flows of elements, ions and abscisic acid in Ricinus communis under potassium limitation. J. Exp. Bot. 53: 241-250.

Saab IN, Sharp RE, Pritchard J, Voetburg GS 1990 Increased endogeneous abscic acid maintains primary root growth and inhibits shoot growth of maize seedlings at low water potential. Plant Physiol. 93: 1329-1336.

Schraut D, Heilmeier H, Hartung W 2005 Radial transport of water and abscisic acid (ABA) in roots of Zea mays under conditions of nutrient deficiency. J. Exp. Bot. 56: 879-886.

Takamure I 1994 Genetic studies on several mutants related to morphological and physiological characters in rice. Mem. Fac. Agr. Hokkaido Univ. 19: 151-202.

Takamure I, Kato K, Kinoshita T 1989 Character expression and gene identification of three mutants responsible for reduced culm number type. Mem. Fac. Agr. Hokkaido Univ. 16: 287-294.

Takamure I, Kinoshita T 1993 Genic analysis of reduced culm number mutants induced by gamma-ray irradiation in rice. Mem. Fac. Agr. Hokkaido Univ. 18: 447-454.

Tang JB, Zeng WY, Wang WM, Ma BT, Liu Y, Li HJ, Xia HG, Li P, Zhu LH 2001 Genetic analysis and gene mapping of a rice few-tillering mutant in early backcross populations (Oryza sativa L.). Sci. China (Ser. C) 3: 208-212.

Ticconi CA, Abel S 2004 Short on phosphate: plant surveillance and countermeasures. Trends Plant Sci. 9: 548-555.

Yasuno N, Takamure I, Kidou S, Tokuji Y, Ureshi A, Funabiki A, Ashikaga K, Yamanouchi U, Yano M, Kato K 2009 Rice shoot branching requires an ATP-binding cassette subfamily G protein. New Phytol. 182: 91-101.

Yasuno N, Yasui Y, Takamure I, Kato K 2007 Genetic interaction between 2 tillering genes, reduced culm numberl ( $\mathrm{rcn} 1)$ and tillering dwarf genes $\mathrm{d} 3$, in rice. J. Hered. 98: 169-172.

Vystoskaya LB, Korobova AV, Kudoyarova GR 2008 Abscisic acid accumulation in the roots of nutrient-limited plants: Its impact on the differential growth of roots and shoots. J. Plant Physiol. 165: 1274-1279. 